\title{
Awareness during anesthesia
}

\author{
Charles H. McLeskey MD
}

$\mathrm{P}$ atient awareness during general anesthesia can occur! Acceptance of this possibility by the competent practicing anesthesiologist is the first step toward its prevention. When addressing audiences on this topic, usually two-thirds of practitioners indicate that at least one patient under their care has experienced unwanted awareness during general anesthesia. The true incidence is likely far greater than we as a community of practitioners recognize.

In a report from England, Aitkenhead and colleagues noted a $0.2 \%$ incidence of spontaneous recall among patients undergoing routine surgery. This frequency of awareness has recently been corroborated by Ranta and colleagues, who reported a $0.4 \%$ incidence of "undisputed awareness" in a study of 2,612 patients from Finland. ${ }^{2}$ In the United States, an incidence of awareness of $0.2 \%$ would represent 30,000 cases of awareness, assuming an annual administration of approximately 15 million general anesthetics. Even higher incidences have been observed during obstetric and cardiac anesthesia where, in general, lower doses of anesthetic agents may be administered. For example, Phillips et al. ${ }^{3}$ reported that $1.1 \%$ of 700 patients who underwent cardiac surgery recalled intraoperative events. Similarly, approximately $1 \%$ of 3,076 patients receiving general anesthesia for Cesarean section remembered something about their operation, although none recalled pain. ${ }^{4}$

Patient awareness during general anesthesia may take the form of implicit or explicit memory. Implicit memory is information retained in the memory that is not accompanied by conscious recollection. Although these patients have no conscious recollection of intraoperative occurrences, they may display symptoms similar to those reported in post-traumatic stress disorder, such as sleep disturbances, dreams, flashbacks and anxiety. The cause of these symptoms, which may persist for months or years, may be uncovered under hypnosis or other forms of detailed psychological testing.

On the other hand, a patient who hears disturbing comments or recalls an unpleasant sensation has demonstrated explicit memory. Auditory perception, the sensation of paralysis and pain were the most frequently mentioned complaints among 26 patients who experienced awareness during general anesthesia. ${ }^{5}$ Similarly, Schwender et al., in a study from Germany, found among 45 patients who displayed explicit memory following general anesthesia that $100 \%$ described an auditory perception, with almost half reporting emotionally relevant remarks. Approximately two-thirds of patients described a tactile sensation (15-20\% reporting pain) while almost $50 \%$ of patients noted a visual perception. ${ }^{6}$

When a patient experiences spontaneous recall of intraoperative events, usually one of the following events has occurred:

1. Anesthetic agents have been intentionally administered in limited quantities for specific clinical reasons. For example, hypovolemic trauma patients, patients undergoing Cesarean section, patients with minimal cardiac reserve, such as those undergoing cardiac surgery may have doses of anesthetic agents intentionally limited.

2. A machine malfunction has resulted in delivery of anesthetic agent in a concentration or dose less than desired or an inadvertent syringe swap has occurred.

3. Apparently adequate quantities of anesthetic agents, at least as judged by blood pressure and other clinical measurements, are delivered to a patient who postoperatively describes awareness. Of the three explanations, this is the most troubling and difficult to comprehend.

Additionally, a small number of patients who complain of intraoperative awareness upon closer examination have confused recall of postoperative events with intraoperative awareness.

\section{Factors affecting frequency}

Although the incidence of awareness as reported in the anesthesia literature has decreased in the past 20 $\mathrm{yr}$, the frequency of patients reporting awareness seems to be increasing. This, I believe, is due to a variety of factors:

1. The increased reliance on muscle relaxants, now utilized in an almost ubiquitous fashion. To the best

Department of Anesthesiology, Medical Director, Perioperative Services, Scott \& White Hospital \& Clinic/Texas A\&M University Health Science Center, Associate Medical Director, Scott \& White Health Plan, Temple, Texas 76508 USA. Phone: 254-724-4528; Fax: 254- 
of my knowledge, there is only one report of awareness in the literature in a spontaneously breathing patient. Perhaps patient movement remains the best premonitory sign of unwanted patient awareness. This useful sign is voided by the administration of a dose of muscle relaxant which produces complete paralysis.

2. Selection of increasingly short-acting anesthetic agents delivered at ever lighter planes of anesthesia. This evolution in our anesthetic practice is designed to provide quick patient recovery, which facilitates room turnover and permits a rapid return to "street fitness" with early discharge home following outpatient surgery. However, it may also increase the risk of awareness.

3. An increased willingness of our patient public to report this complication.

\section{Efforts at prevention developing}

Until the reasons for awareness are more clearly elucidated and/or a monitor is developed to indicate reliably the circumstances when patient awareness may be occurring, efforts at prevention remain largely untested and in a primitive state of development. Nevertheless, the following general suggestions represent an expanded discussion of possible techniques mentioned in a comprehensive review by Ghoneim and Block.?

1. Consider the use of anesthetic agents with particular amnestic qualities, such as a benzodiazepine or scopolamine, given either as a premedicant or a supplement to general anesthesia. Relying on benzodiazepines, however, may give a false sense of security since these agents have a broad range of effectiveness in various patients. The use of scopolamine or ketamine in a hemodynamically unstable, compromised trauma patient may be useful though certainly far from $100 \%$ effective in providing amnesia.

2. Muscle relaxants should be avoided unless indicated for surgery and then, if clinically appropriate, they should be administered in a dose that does not produce complete paralysis. By avoiding complete paralysis, patient movement may serve as a crude indicator of potential awareness.

3. Administer inhalational agents at a minimum end-tidal concentration of 0.8 MAC. Dwyer, et al. ${ }^{8}$ found that end-tidal concentrations of isoflurane at 0.6 MAC prevented conscious recall in a series of 45 patients prior to undergoing surgery. However, there is no accurate information about the MAC fraction that will "guarantee" unconsciousness during surgery in virtually all patients. Equi-MAC concentrations of different inhalational agents and nitrous oxide may not be equal in preventing awareness. For example, isoflurane has been shown to be more potent than equi-MAC concentrations of nitrous oxide in preventing memory and awareness. ${ }^{9}$ Nevertheless, review of published figures on awareness related to various concentrations of nitrous oxide, combined with extrapolation of standard deviation curves, suggests that a minimum value likely to prevent awareness is approximately $0.8 \mathrm{MAC}$.

4. Perform a meticulous check of the anesthesia machine. Assuring that an anesthesia machine is delivering what is sought affords confidence that fewer patients will suffer awareness. In addition, monitoring, the end-tidal concentration of inhalational agents provides a further check that inhalational agents are being delivered as desired. Recently, a malpractice claim of awareness that was settled for $\$ 150,000$ resulted when an empty inhalational agent vaporizer was undetected by the anesthesiologist. Vigilance during the course of an anesthetic includes ascertaining continuing proper machine functioning.

5. Supplemental doses of induction agents should be considered when a difficult intubation is accompanied by a protracted laryngoscopy.

6. In non-hypoxic mixtures, nitrous oxide alone may be insufficient to guarantee lack of awareness. Opioids in conventional doses are not anesthetic agents. Thus, nitrous oxide/opioid-based anesthetic techniques should be supplemented with a potent inhalational agent or infusion of other intravenous agents, which enhance the likelihood of amnesia.

7. Discourage remarks made about an anesthetized patient. Some comments regarding unexpected surgical findings or remarks about the patient's body weight have been recalled verbatim. ${ }^{3}$ Cognitive processing of auditory information may occur during apparently adequate levels of anesthesia. It is possible that an unconscious mind may register negative or traumatic comments during an anesthetic maintained at a depth which is otherwise adequate for surgery. If recalled, derogatory remarks may be aggressively litigated by the offended patient; if not recalled, these remarks may result in psychological damage.

8. Consider offering informed consent to patients regarding the possibility of awareness, especially if awareness is reasonably foreseeable. As noted earlier, circumstances where awareness is more likely include patients in whom we anticipate administering an intentionally light level of anesthesia, e.g., trauma patients, those undergoing cesarean section or cardiac surgery. Certainly, informed consent should be offered if a patient specifically requests information regarding this issue. McCleane and Cooper have noted that $50 \%$ of patients express concern preopera- 
tively that they may not be asleep during an operation, and more than $25 \%$ were concerned postoperatively about being adequately asleep during a future operation, even though no awareness or recall had been experienced during their current procedure. ${ }^{10}$

Although controversial, some authors have indicated that suggestions made to and retained by a patient may be used to advantage. Bonke $e t$ al., found that patients older than $55 \mathrm{yr}$ undergoing biliary tract surgery who were given positive suggestions intraoperatively had a shorter postoperative stay. ${ }^{11}$ Evans and Richardson similarly found that positive intraoperative suggestions shortened time to hospital discharge in patients undergoing abdominal hysterectomy. ${ }^{12}$ Others have proposed that positive intraoperative suggestions may result not only in earlier ambulation but also in reduced postoperative nausea and pain and improved wound healing. However, other investigators have been unable to confirm these beneficial effects of therapeutic suggestions. ${ }^{13,14}$

\section{Medicolegal considerations}

Efforts at prevention are of even greater importance since patient awareness during general anesthesia carries with it a medicolegal consideration. William $M$. Gild MB, JD, in the June 1993 issue of the $A S A$ Newsletter, reviewed 2,400 claims in the ASA Closed Claims Project and found that approximately $2 \%$ of all claims were related to instances of patient awareness. In the June 1996 issue of the ASA Newsletter, Karen Domino $M D$, reported a similar incidence among 3,533 reviewed claims. Further, she noted an increased size of damage for this claim, with the highest award now standing at $\$ 600,000.00$. Judging from the claims of which I have been recently made aware, I believe the incidence of medicolegal action regarding unwanted awareness during anesthesia is increasing dramatically.

\section{A new monitor}

Until recently, there was no monitor available that was reliable in letting us know at any given moment in time if a patient had been rendered in an anesthetic state where memory and recall were prevented. Although several new such monitors are under study, one known as the BIS monitor (Bispectral Index, Aspect Medical Systems,Natick, MA), has undergone extensive investigation and is approved for use. This monitor is a processed EEG with advanced algorithms that report a number from 0 to 100: 100 represents an awake state and 0 represents complete EEG inactivity. The monitoring electrodes are simple and quick to apply. Several studies describe high correlation of the measured BIS parameter to the state of consciousness or the state unconsciousness with lack of free recall. ${ }^{15}$ This is in contradistinction to using vital signs which, at best, represent only a crude form of determining the state of consciousness or unconsciousness. ${ }^{16}$ Early data confirm that the BIS monitor is useful in determining patients who may be a lighter level of consciousness than desired (and thus at potential risk of awareness) and also those who are at a deeper level of unconsciousness than ideal (and thus likely to experience an emergence that is slower than necessary). ${ }^{17}$

\section{Raising our sensitivity to the issue of "awareness"}

Until supplemental CNS monitoring devices are in wide use, we must accept the fact that we never know for sure if a patient is at an anesthetic depth that prevents awareness and recall. In this circumstance, the most effective technique for reducing patient awareness is an increased level of sensitivity among anesthesia colleagues and other health care providers to this potential problem. Educated surgeons, nurses and other practitioners who recognize the entity of awareness may inform anesthesia providers should a patient complain postoperatively of recall.

It is essential that all healthcare providers behave in a considerate fashion when coming in contact with anesthetized patients. Words and sentiments should be selected that are reassuring to the patient, should they be heard, rather than those that would be deemed traumatic, alarming or disrespectful. The practice of anesthesiology requires careful timing and selection of agents to provide a proper anesthetic depth that permits rapid emergence and recovery while yet assuring amnesia. In this context, Ted Eger suggests, "Not only must we not give too much anesthesia, we must not give too little".

\section{References}

1 Liu D, Thorp S, Aitkenbead AR. Incidence of awareness with recall during general anaesthesia. Anaesthesia 1991; 46: 435-7.

2 Ranta SO, Laurila $R$, Saario J, et al. Awareness with recall during general anesthesia: incidence and risk factors. Anesth Analg 1998; 86: 1084-9.

3 Phillips AA, McLean RF, Devitt JH, et al. Recall of intraoperative events after general anaesthesia and cardiopulmonary bypass. Can J Anaesth 1993; 40: 922-6.

4 Lyons $G$, MacDonald $R$. Awareness during Caesarean section. Anaesthesia 1991; 46: 62-4.

5 Moerman $M$, Bonke B, Oosting J. Awareness and recall during general anesthesia. Anesthesiology 1993; 79 : 454-64. 
6 Schwender D, Kunze-Kronawitter $H$, Dietrich P, et al. Conscious awareness during general anaesthesia: patients' perceptions, emotions, cognition and reactions. Br J Anaesth 1998; 80: 133-9.

7 Ghoneim MM, Block RI. Learning and consciousness during general anesthesia. Anesthesiology 1992; 76: 279-305.

8 Dwyer R, Bennett $H L$, Eger EI, et al. Isoflurane anesthesia prevents unconscious learning. Anesth Analg 1992; 75: 107-12.

9 Dwyer R, Bennett, HL Eger EI, et al Effects of isoflurane and nitrous oxide in subanesthetic concentrations on memory and responsiveness in volunteers. Anesthesiology 1992; 77: 888-98.

10 McCleane GJ, Cooper $R$. The nature of perioperative anxiety. Anaesthesia 1990; 45: 153-5.

11 Bonke S, Schmitz PIM, Verbages $F$, et al. Clinical study of so-called unconscious perception during general anaesthesia. Br J Anaesth. 1986; 58: 957-64.

12 Evans $C$, Richardson $P H$. Improved recovery and reduced postoperative stay after therapeutic suggestions during general anesthesia. Lancet 1988; 2: 49l-3.

13 Boeke S, Bonke B, Bouwbuis-Hoogerwerf $M L$, et al. Effects of sounds during anaesthesia on postoperative course. Br J Anaesth 1988; 60: 697-702.

14 Liu HD, Standen PJ, Aitkenhead AR. Therapeutic suggestions during general anaesthesia in patients undergoing hysterectomy. Br J Anaesth 1992; 68: 277-81.

15 Glass, PS, Bloom M, Kearse $L$, et al. Bispectral analysis measures sedation and memory effects of propofol, midazolam, isoflurane and alfentanil in healthy volunteers. Anesthesiology 1997; 86: 836-47.

16 Flaishon R, Windsor A, Sigl J, et al. Recovery of consciousness after thiopental or propofol. Anesthesiology 1997; 86: 613-9.

17 Payne FB, Sebel PS, Glass PS, et al. Bispectral index (BIS) monitoring allows faster emergence from propofol, alfentanil / $\mathrm{n}_{20}$ anesthesia. Anesthesiology 1996; 85: A1056. 


\title{
La conscience pendant l'anesthésie
}

\author{
Charles H. McLeskey MD
}

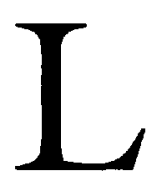

E patient peut être conscient sous anesthésie générale! Accepter cette possibilité, c'est le début de sa prévention. Quand on aborde ce sujet devant un auditoire de praticiens, les deux tiers d'entre eux habituellement mentionnent qu'au moins un patient sous leurs soins a subi des épisodes de conscience non souhaitée pendant l'anesthésie générale. La véritable incidence de ce phénomène est beaucoup plus élevée que ce que nous reconnaissons.

Dans un article publié en Angleterre, Aitkenhead et ses collègues ont noté une incidence de $0,2 \%$ d'évocation spontanée parmi les patients qui subissent une intervention chirurgicale de routine. Cette fréquence d'évocation a été récemment corroborée par Ranta et ses collaborateurs qui rapportent une incidence de 0,4 $\%$ de «conscience incontestable» dans une étude de 2 612 patients de Finlande. ${ }^{2}$ Aux États-Unis, une incidence de conscience de $0,2 \%$ représenterait 30000 cas d'évocation, en supposant l'administration annuelle d'environ quinze millions d'anesthésies générales. On a même observé des incidences plus élevées pendant des interventions obstétricales et cardiaques où, en général, on peut administrer des doses plus faibles d'anesthésiques. Par exemple, Phillips et coll. ${ }^{3}$ rapportent que $1,1 \%$ des 700 patients qui ont subi une intervention cardiaque se souviennent d'événements peropératoires. De même, environ $1 \%$ des 3076 patientes qui ont reçu une anesthésie générale pour une césarienne se rappellent de leur opération, bien qu'aucune ne se souvienne de douleurs."

La conscience du patient pendant l'anesthésie générale peut présenter la forme d'une évocation implicite ou explicite. L'évocation implicite est une information retenue dans la mémoire, mais qui n'est pas accompagnée de souvenir conscient. Bien que ces patients n'aient pas de souvenir conscient de faits peropératoires, ils peuvent présenter des symptômes semblables à ceux du syndrome de stress post-traumatique, comme les troubles du sommeil, les rêves, les retours en arrière et l'anxiété. La cause de ces symptômes, qui peuvent durer des mois ou des années, peut être découverte sous hypnose ou d'autres formes d'évaluation phychologique détaillée.

Par contre, un patient qui entend des commentaires troublants ou se rappelle des sensations désagréables a démontré une mémoire explicite. La perception auditive, la sensation de paralysie et de douleur sont les plaintes le plus souvent formulées parmi 26 patients qui ont été conscients pendant l'anesthésie générale. ${ }^{5}$ Schwender et coll., à partir d'une étude faite en Allemagne, ont trouvé, parmi 45 patients qui ont affiché une mémoire explicite après une anesthésie générale, que $100 \%$ ont décrit une perception auditive, et presque la moitié d'entre eux ont rapporté des remarques émotivement significatives. Environ les deux tiers ont décrit une sensation tactile (15-20\% ont mentionné de la douleur) tandis que $50 \%$ des patients ont noté une perception visuelle. ${ }^{6}$

Quand un patient a le souvenir spontané d'événements peropératoires, l'un des incidents suivants s'est habituellement produit :

1. Les anesthésiques ont été intentionnellement administrés en quantité limitée pour des raisons spécifiques. Par exemple : trauma hypovolémique, césarienne, réserve cardiaque minimale. Ceux qui subissent une intervention cardiaque peuvent recevoir des doses d'anesthésiques intentionnellement limitées.

2. Le dérèglement d'une machine a provoqué la livraison d'anesthésique selon une concentration ou une dose moindre que celle qui avait été prévue ou un échange malencontreux de seringue s'est produit.

3. Des quantités en apparence exactes d'anesthésiques, au moins si l'on en juge par la tension artérielle et les autres mesures cliniques, sont administrées à un patient qui décrit, après l'opération, des épisodes de conscience. Des trois explications, cette dernière est la plus troublante et difficile à comprendre.

De plus, un petit nombre de patients qui se plaignent de conscience peropératoire, révèlent en fait, après un examen plus approfondi, des souvenirs confus d'événements postopératoires perçus comme des événements peropératoires.

\section{Les facteurs de fréquence}

Bien que l'incidence de conscience tel que rapportée dans la littérature anesthésique ait diminué pendant les vingt dernières années, la fréquence des incidents rapportés par les patients semble augmenter. Ce qui, je crois, dépend d'une variété de facteurs :

1. Le recours croissant aux myorelaxants, qui sont presque omniprésents. Que je sache, il n'y a qu'un seul 
rapport d'état de conscience dans la littérature chez un patient qui respire spontanément. Le mouvement du patient demeure sans doute le meilleur signe annonciateur d'un état de conscience non voulu. Ce signe utile est mis de côté par l'administration d'une dose de myorelaxant qui produit une paralysie complète.

2. La sélection des anesthésiques d'action de plus en plus brève administrés à des niveaux plus légers de profondeur d'anesthésie. Cette évolution de notre pratique a été conçue pour permettre une récupération rapide, ce qui facilite la rotation des patients dans l'hôpital et permet un retour rapide aux activités régulières et un congé précoce à la suite d'une intervention ambulatoire. Cependant, cela peut aussi augmenter le risque du retour de la conscience.

3. L'empressement plus grand de nos patients à rapporter cette complication.

\section{Les efforts de prévention}

Jusqu'à ce que les raisons de l'état de conscience soient plus clairement élucidées et/ou qu'un moniteur soit mis au point pour indiquer de façon fiable les occasion où la reprise de conscience pourrait survenir, les efforts de prévention demeurent largement non testés et dans un état primitif de développement. Néanmoins, les suggestions générales qui suivent représentent un exposé élargi des techniques possibles mentionnées par Ghoneim et Block dans un article détaillé. ?

1. Envisager l'utilisation d'anesthésiques aux qualités amnésiantes particulières comme une benzodiazépine ou une scopolamine, administrée comme une prémédication ou un supplément à l'anesthésie générale. Se fier aux benzodiazépines peut toutefois procurer une fausse sécurité, puisque ces agents ont un large éventail d'efficacité chez différents patients. L'utilisation de scopolamine ou de kétamine dans un cas d'instabilité hémodynamique et de trauma à risques élevés peut être utile, quoique la qualité de l'amnésie produite sera très en deçà de l'efficacité maximale.

2. Les myorelaxants devraient être évités à moins d'être indiqués pour une intervention et, dans ce cas, si c'est approprié, ils devraient être administrés en doses qui ne produisent pas de paralysie complète. En évitant la paralysie complète, le mouvement possible du patient peut servir d'indicateur précis d'un état de conscience potentiel.

3. Administrer des agents d'inhalation à des concentrations de fin d'expiration minimales de 0,8 CAM. Dwyer, et coll. ${ }^{8}$ ont trouvé que les concentrations de fin d'expiration d'isoflurane à 0,6 CAM prévenaient le rappel conscient dans une série préopératoire de $\mathbf{4 5}$ patients. Cependant, il n'y a pas d'information exacte sur la fraction de CAM qui pourrait «garantir» l'inconscience pendant l'intervention chez pratiquement tous les patients. Les concentrations équi-CAM de différents agents d'inhalation et du protoxyde d'azote peuvent être inégales à prévenir l'état de conscience. Par exemple, on a montré que l'isoflurane était plus puissant que les concentrations équi-CAM de protoxyde d'azote pour empêcher la mémoire et la conscience de se manifester. ${ }^{9}$ Néanmoins, la révision des chiffres publiés sur l'état de conscience reliée à diverses concentrations de protoxyde d'azote, combinée à l'extrapolation des courbes d'écart type, avance que la valeur minimale qui pourrait prévenir l'état de conscience serait d'environ $0,8 \mathrm{MAC}$.

4. Faire une vérification méticuleuse de la machine à anesthésie. S'assurer que la machine livre ce qu'on désire nous permet de croire que peu de patients vont vivre une reprise de conscience. De plus, le monitorage de la concentration de fin d'expiration des agents d'inhalation fournit une preuve supplémentaire que le médicament est administré comme on le souhaite. Récemment, une réclamation pour faute professionnelle a été réglée à $150000 \$$. Un vaporisateur d'agent d'inhalation vide n'avait pas été détecté par l'anesthésiologiste. La vigilance pendant le déroulement de l'anesthésie inclut de s'assurer du bon fonctionnment mécanique continu.

5. Des doses supplémentaires d'agents d'induction devraient être envisagées quand une intubation difficile s'accompagne d'une laryngoscopie prolongée.

6. Dans les mélanges non hypoxiques, le protoxyde d'azote seul pourrait être insuffisant pour garantir l'inconscience. Les opiö̈des en doses traditionnelles ne sont pas des agents anesthésiques. Donc, les régimes anesthésiques à base d'un mélange de protoxyde d'azote et d'opioïde devrait être complétés par un agent d'inhalation puissant ou une perfusion d'un autre agent intraveineux qui augmente la possibilité d'amnésie.

7. Décourager les remarques sur un patient sous anesthésie. Certains commentaires portant sur des constatations chirurgicales inattendues ou des remarques au sujet du poids du patient ont été répétées textuellement. ${ }^{3}$ Le traitement cognitif d'informations auditives peut survenir pendant une anesthésie apparemment adéquate. Il est possible qu'un esprit inconscient puisse enregister des commentaires négatifs ou traumatisants pendant une anesthésie maintenue à une profondeur par ailleurs suffisante pour l'opération. Si elles sont évoquées, les remarques désobligeantes peuvent être agressivement mises en litige par le patient offensé; si elles ne sont pas évoquées, ces remarques peuvent entraîner des troubles psychologiques. 
8. Envisager d'obtenir le consentement éclairé du patient en regard de la possibilité d'un retour de la conscience, surtout si cette éventualité est raisonnablement prévisible. Comme on l'a mentionné déjà, les circonstances où la reprise de conscience est la plus probable comprennent les cas de patients chez qui nous prévoyons administrer un niveau intentionnellement plus bas d'anesthésie, comme dans les cas de trauma, de césarienne ou d'intervention cardiaque. Il est certain qu'un consentement éclairé devrait être obtenu si un patient demande des informations précises à ce sujet. McCleane et Cooper ont noté que $50 \%$ des patients expriment des inquiétudes avant l'opération quant au fait possible de n'être pas endormi pendant l'intervention, et plus de $\mathbf{2 5} \%$ sont préoccupés après l'opération par la possibilité d'être conscient lors d'une future opération, même s'ils ne se souviennent de rien à la suite de l'intervention actuelle. ${ }^{10}$

Quoique controversé, certains auteurs ont indiqué que des suggestions faites à un patient et retenues par lui peuvent être un avantage. Bonke et coll., ont trouvé que des patients de plus de $\mathbf{5 5}$ ans subissant une opération des voies biliaires et à qui on avait présenté des suggestions positives peropératoires ont eu un séjour postopératoire plus court. ${ }^{11}$ Evans et Richardson ont aussi découvert que l'usage de suggestions peropératoires positives permettait de réduire le séjour postopératoire de patientes subissant une hystérectomie abdominale. ${ }^{12}$ D'autres ont proposé que ces suggestions pourraient non seulement favoriser la déambulation plus hâtive, mais réduiraient aussi les nausées et la douleur postopératoires et amélioreraient la cicatrisation. Cependant, d'autres chercheurs n'ont pu confirmer les effets de ces suggestions thérapeutiques.

\section{Considérations médico-légales}

Les efforts de prévention sont d'autant plus importants depuis que l'apparition de la conscience pendant l'anesthésie générale comporte un aspect médico-légal. William M. Gild MB, JD, dans le numéro de juin 1993 du $A S A$ Newsletter, a examiné 2400 réclamations du ASA Closed Claims Project (réclamations réglées) et a trouvé qu'environ $2 \%$ de toutes les réclamations étaient reliées à des cas de patients conscients pendant l'anesthésie. Dans le numéro de juin 1996 du $A S A$ Newsletter, Karen Domino MD, a rapporté une incidence similaire parmi 3533 réclamations révisées. De plus, elle a noté l'importance croissante des dommages mentionnés dans la réclamation et le montant le plus élevé qui est maintenant de $600000 \$$. Si j'en juge d'après les réclamations dont $j$ 'ai pris connaissance récemment, je crois que l'incidence d'action médico- légale concernant l'état de conscience non désiré pendant l'anesthésie s'accroît de façon importante.

\section{Un nouveau moniteur}

Jusqu'à récemment, il n'existait pas de moniteur fiable nous permettant de savoir à n'importe quel moment de l'intervention si un patient avait reçu suffisamment d'anesthésique pour empêcher l'état de conscience et le souvenir de se manifester. Bien que des nouveaux moniteurs semblables soient à l'étude, l'un d'eux, le moniteur BIS (Bispectral Index, Aspect Medical Systems, Natick, MA), a été étudié plus attentivement et son utilisation est maintenant approuvée. Ce moniteur est un EEG traité avec des algorithmes perfectionnés qui rapportent des nombres entre 0 et 100 : 100 représente l'état d'éveil et 0 représente l'inactivité EEG totale. Les électrodes du moniteur sont faciles et rapides à appliquer. Certaines études décrivent d'importantes corrélations entre les paramètres de BIS mesurés et l'état de conscience ou entre l'état d'inconscience et l'absence de souvenir spontané. ${ }^{15}$ Cela arrive en contradiction avec l'usage des signes vitaux qui, au mieux, ne représentent qu'une forme grossière de détermination de l'état de conscience ou d'inconscience. ${ }^{16}$ Les données préliminaires confirment que le BIS est utile pour désigner les patients qui peuvent être à un degré d'inconscience plus léger que désiré (et, donc, à risque potentiel de conscience) et aussi ceux qui sont à un degré plus profond d'inconscience que l'idéal souhaité (et, donc, à risque d'un réveil plus lent que nécessaire). ${ }^{17}$

\section{Augmenter notre sensibilité à l'état de "conscience»}

Jusqu'à ce que des moniteurs du SNC supplémentaires soient d'usage répandu, nous devons accepter le fait de ne jamais savoir de façon certaine si un patient a reçu une anesthésie assez profonde pour qu'il n'ait ni conscience ni souvenir de son opération. Dans ces circonstances, la technique la plus efficace de réduction de l'état de conscience chez le patient est d'augmenter la sensibilité de nos collègues anesthésistes et autres soignants face à ce problème potentiel. Des chirurgiens, des infirmières et d'autres praticiens qui reconnaissent cette problématique peuvent informer ceux qui ont administré l'anesthésie lorsqu'un patient dit se souvenir de son opération.

Il est essentiel que le personnel soignant agisse avec certains égards pour les patients qui reçoivent de l'anesthésie. Les mots et les sentiments exprimés doivent être choisis pour rassurer le patient, si ces mots peuvent être entendus, plutôt que de traumatiser, d'alarmer ou de manquer de respect. La pratique de l'anesthésie exige un certain ordre d'utilisation et 
une sélection des médicaments pour fournir une profondeur d'anesthésie appropriée qui permet le réveil rapide et la récupération tout en assurant l'amnésie. Dans ce contexte, Ted Eger suggère, "Non seulement, nous ne devons pas administrer trop d'anesthésie, nous ne devons pas en administrer trop peu».

Références

(Voir page R82) 